\title{
Verificação da preditibilidade de falhas em sistemas a eventos discretos com linguagem não viva e com ciclos de estados conectados somente por eventos não observáveis *
}

\author{
Raphael J. Barcelos* Maurício A. Corrêa ${ }^{* *}$ João C. Basilio* \\ * COPPE - Programa de Engenharia Elétrica, Universidade Federal do \\ Riode Janeiro,RJ (e-mail: raphajulio@gmail.com,basilio@dee.ufrj.br) \\ ** Escola Politécnica - Engenharia Elétrica, Universidade Federal do \\ Rio de Janeiro, RJ (e-mail: mauricioaugusto@poli.ufrj.br).
}

\begin{abstract}
:
Predictability is a discrete event system (DES) property that aims to foresee some specific event occurrence, usually seen as failures. Due to common restrictive assumptions, such as nonexistence of cycles of states connected with unobservable events only and language liveness, the existing methods for predictability verification do not include some classes of DES with more general behavior. In this paper, we revisit the problem of predictability in a more general scenario, in which, we remove the above assumption, and present a diagnoser-based algorithm for its verification.

Resumo: A preditibilidade é uma propriedade de sistemas a eventos discretos (SED) que objetiva prever a ocorrência de eventos específicos, geralmente referidos como falhas. Devido a hipóteses restritivas, tais como ausência de ciclos de estados conectados somente por eventos não observáveis e que a linguagem seja viva, esses métodos não abrangem algumas classes de SEDs com comportamentos mais gerais. Neste artigo, o problema de preditibilidade é revisitado em um contexto mais geral, no qual as hipóteses acima são removidas, além de propor um algoritmo para a sua verificação.
\end{abstract}

Keywords: Discrete event systems; automaton; predictability; failure prognosis; predictability verification.

Palavras-chaves: Sistema a eventos discretos; autômatos; preditibilidade; prognose de falhas; verificação de preditibilidade.

\section{INTRODUÇÃO}

A preditibilidade, também referida como prognose, é uma propriedade de sistemas a eventos discretos (SEDs), cuja primeira definição foi apresentada em Genc e Lafortune (2009) e visa antever a ocorrência de falhas em um sistema.

Kumar e Takai (2010) abordam a preditibilidade em uma arquitetura descentralizada, enquanto que em Takai e Kumar (2012) é realizada uma abordagem em arquitetura distribuída por meio de atrasos e limitações nas comunicações envolvendo tanto a planta quanto os preditores. Khoumsi e Chakib (2012) interpretam a arquitetura descentralizada apresentada em Kumar e Takai (2010) como sendo uma arquitetura disjuntiva, propõem uma arquitetura conjuntiva e também uma arquitetura mista, que combina e generaliza as estruturas disjuntivas e conjuntivas. Khoumsi e Chakib (2009) também generalizam a preditibilidade em arquite-

\footnotetext{
* Este trabalho foi financiado em parte pela Coordenação de Aperfeiçoamento de Pessoal de Nível Superior (CAPES), Código de Financiamento 001, e pelo Conselho Nacional de Desenvolvimento Científico e Tecnológico (CNPq), processo número 309652/2017-0.
}

turas conjuntivas e disjuntivas por meio de um preditor multi-decisão.

O conceito de preditibilidade também é explorado em outros contextos como, por exemplo, por Yin e Li (2016a), que avaliam o tempo de resposta após a detecção de falha por preditores, Yin e Li (2016b), que abordam perdas na transmissão de informação enviadas pelos preditores a um coordenador, Takai (2015), que verifica a preditibilidade na presença de deadlocks e Chen e Kumar (2015), que abordam a preditibilidade em sistemas estocásticos.

Em relação à diagnosticabilidade, um problema similar à preditibilidade no sentido de que ambos lidam com falhas em sistemas e eventos discretos, Jiang e Kumar (2004) afirmam que a preditibilidade é uma condição necessária para a diagnosticabilidade. Em contrapartida, Genc e Lafortune (2009) afirmam que, sob suas hipóteses restritivas, a preditibilidade é uma condição suficiente para a diagnosticabilidade. Kumar e Takai (2010) apresentam que, em contextos mais gerais, preditibilidade e diagnosticabilidade, tanto em arquiteturas centralizadas quanto descentralizadas, são problemas incomparáveis. 
Neste trabalho, aborda-se preditibilidade por meio de autômatos. Nesse sentido, a fim de lidar com sistemas mais gerais, será proposta uma nova ferramenta inspirada em Viana e Basilio (2019) para verificar a preditibilidade de falhas que não requer as hipóteses restritivas apresentadas em Genc e Lafortune (2009) e Liu (2019) (linguagem viva e ausência de ciclos de estados conectados somente por eventos não observáveis).

Embora existam trabalhos que realizem a verificação de preditibilidade via verificadores, este trabalho utilizará um autômato teste para a verificação, que é baseado em diagnosticadores, e apresentará um algoritmo para construção do autômato teste. O uso de diagnosticadores é justificado por Clavijo e Basilio (2017), em que é mostrado que, embora o diagnosticador possua mais estados que o verificador, o primeiro possui, em média, menos estados.

Este artigo é dividido da seguinte forma. A Seção 2 apresenta os conceitos básicos sobre SEDs e preditibilidade. A Seção 3 apresenta a motivação do problema para que seja proposta uma nova estratégia para verificação de preditibilidade. A Seção 4 propõe uma estratégia para a sua verificação, assim como um algoritmo. A Seção 5 resume todas as contribuições do trabalho e também sugere possíveis extensões desta pesquisa.

\section{FUNDAMENTOS TEÓRICOS}

Autômatos de estados finitos são o formalismo aqui utilizado para modelar SEDs. Um autômato $G$ é definido pela sêxtupla $G:=\left(X, \Sigma, f, \Gamma, x_{0}, X_{m}\right)$, em que $X$ é o conjunto finito de estados, $\Sigma$ é o conjunto finito de eventos, $f: X \times \Sigma \rightarrow X$ é a função de transição, $\Gamma: X \rightarrow 2^{\Sigma}$, definido por $\Gamma(x):=\{\sigma \in \Sigma: f(x, \sigma) !\}$, é o conjunto de eventos ativos, em que $f(x, \sigma)$ ! significa que $f(x, \sigma)$ é definido, $x_{0} \in X$ é o estado inicial e $X_{m} \subseteq X$ é o conjunto de estados marcados (Cassandras e Lafortune, 2008). A função de transição pode ser estendida para $f: X \times \Sigma^{*} \rightarrow X\left(\Sigma^{*}\right.$ representa o Fecho-de-Kleene de $\Sigma)$, pela recursão $f(x, \epsilon)=x ; f(x, s \sigma)=f(f(x, s), \sigma)$; $s \in \Sigma^{*}, \sigma \in \Sigma$. A linguagem gerada por $G$ é definida como $\mathcal{L}(G):=\left\{s \in \Sigma^{*}: f\left(x_{0}, s\right)\right.$ ! $\}$ e será denotada por $L$. Supõe-se que o conjunto de eventos $\Sigma$ seja particionado em $\Sigma=\Sigma_{o} \dot{\cup} \Sigma_{u o}$, em que $\Sigma_{o}$ e $\Sigma_{u o}$ são, respectivamente, o conjunto de eventos observáveis e não observáveis.

Ao longo do texto, o fecho do prefixo de uma sequência $s$ será representado por $\bar{s}$, sendo definido como $\bar{s}:=\{u \in$ $\left.\Sigma^{*}:\left(\exists v \in \Sigma^{*}\right)[u v=s]\right\}$. A pós-linguagem de $L$ após uma sequência $s$ é definida por $L / s:=\left\{t \in \Sigma^{*}:\right.$ st $\left.\in L\right\}$. Uma linguagem $L$ é dita viva se $\forall s \in L, \exists \sigma \in \Sigma: s \sigma \in L$. Dados dois conjuntos de eventos, $\Sigma_{a}$ e $\Sigma_{b}$, em que $\Sigma_{b} \subseteq \Sigma_{a}$, a projeção natural, ou apenas projeção, é definida por $P_{a, b}: \Sigma_{a}^{*} \rightarrow \Sigma_{b}^{*}$, em que $P_{a, b}(\varepsilon)=\varepsilon$, sendo $\varepsilon$ a sequência nula; $P_{a, b}(\sigma)=\sigma$ se $\sigma \in \Sigma_{b} ; P_{a, b}(\sigma)=\varepsilon$ se $\sigma \in \Sigma_{a} \backslash \Sigma_{b}$, e; $P_{a, b}(s \sigma)=P_{a, b}(s) P_{a, b}(\sigma)$, em que $s \in \Sigma_{a}^{*}, \sigma \in \Sigma_{a}$. A projeção inversa é definida por $P_{a, b}^{-1}: \Sigma_{b}^{*} \rightarrow \Sigma_{a}^{*}$, em que $P_{a, b}^{-1}(t):=\left\{s \in \Sigma_{a}^{*}: P_{a, b}(s)=t\right\}$. O fecho do prefixo, a projeção e a projeção inversa são estendidas sobre uma linguagem $L$ aplicando-as a todas as sequências de $L$.

A operação parte acessível de um autômato remove todos os estados que não são alcançados a partir do estado inicial por meio de sequências, e é definida por
$A c(G):=\left(X_{A c}, \Sigma, f_{A c}, \Gamma_{A c}, x_{0}, X_{A c, m}\right)$, em que $X_{A c}:=$ $\left\{x \in X:\left(\exists s \in \Sigma^{*}\right)\left[f\left(x_{0}, s\right)=x\right]\right\}, f_{A c}: X_{A c} \times$ $\Sigma^{*} \rightarrow X_{A c}$ em que $f_{A c}(x, \sigma)=f(x, \sigma)$ se $f(x, \sigma) \in X_{A c}$, e não definida, caso contrário, e $X_{A c, m}=X_{m} \cap X_{A c}$. A composição paralela entre autômatos cria um novo autômato em que o comportamento comum dos autômatos é sincronizado e seus comportamentos particulares ocorrem livremente, sendo formalmente definida por $G_{1} \| G_{2}:=$ $A c\left(X_{1} \times X_{2}, \Sigma_{1} \cup \Sigma_{2}, f_{1|| 2}, \Gamma_{1|| 2}, x_{0,1} \times x_{0,2}, X_{m, 1} \times X_{m, 2}\right)$, em que $f_{1|| 2}\left(\left(x_{1}, x_{2}\right), \sigma\right):=\left(f_{1}\left(x_{1}, \sigma\right), x_{2}\right)$ se $\sigma \in \Gamma_{1}\left(x_{1}\right) \backslash$ $\Sigma_{2} ;\left(x_{1}, f_{2}\left(x_{2}, \sigma\right)\right)$ se $\sigma \in \Gamma_{2}\left(x_{2}\right) \backslash \Sigma_{1} ;\left(f_{1}\left(x_{1}, \sigma\right), f_{2}\left(x_{2}, \sigma\right)\right)$ se $\sigma \in \Gamma_{1}\left(x_{1}\right) \cap \Gamma_{2}\left(x_{2}\right)$, e não definida, caso contrário.

O alcance não observável é definido por $U R\left(x, \Sigma_{o}\right):=$ $\left\{x^{\prime} \in X:\left(\exists s \in \Sigma_{u o}^{*}\right)\left[f(x, s)=x^{\prime}\right]\right\}$. Sua extensão para um conjunto de estados $Y \in 2^{X}$ é dada por: $U R\left(Y, \Sigma_{o}\right):=\cup_{x \in Y} U R\left(x, \Sigma_{o}\right)$. Dessa forma, o observador do autômato $G$ é dado por $G_{o b s}=O_{b s}\left(G, \Sigma_{o}\right):=$ $\left(X_{o b s}, \Sigma_{o}, f_{o b s}, \Gamma_{o b s}, x_{0, o b s}, X_{o b s, m}\right)$, em que $X_{o b s} \in 2^{X}$, $f_{o b s}\left(x_{o b s}, \sigma\right):=\bigcup_{x \in x_{o b s} \wedge f(x, \sigma) !} U R\left(f(x, \sigma), \Sigma_{o}\right), \Gamma_{o b s}\left(x_{o b s}\right)$ $:=\bigcup_{x \in x_{o b s}} \Gamma(x) \cap \Sigma_{o}, x_{0, o b s}:=U R\left(x_{0}, \Sigma_{o}\right)$. Finalmente, $\mathcal{L}\left(G_{o b s}\right) \stackrel{=}{=} P_{o}\left(\mathcal{L}\left(G_{o b s}\right)\right)$ será denotado por $L_{o b s}$, em que $P_{o}: \Sigma^{*} \rightarrow \Sigma_{o}^{*}$.

Seja $\Sigma_{f} \subset \Sigma_{u o}$ o conjunto formado pelos eventos de falha, o qual será neste artigo suposto unitário sem perda de generalidade, i.e., $\Sigma_{f}=\left\{\sigma_{f}\right\}$. A diagnose de falhas é uma propriedade de SEDs que visa saber se toda ocorrência de falha será eventualmente detectada pelo diagnosticador, isto é, sempre que o evento de falha $\sigma_{f}$ ocorrer, ele será detectado após um número finito de observações.

Representa-se por $\Psi(\sigma)$ o conjunto composto por todas as sequências de $L=L(G)$ que terminam com o evento $\sigma$, formalmente definido por $\Psi(\sigma):=\left\{s \sigma \in L: s \in \Sigma^{*}, \sigma \in\right.$ $\Sigma$ \}, cujo conceito pode ser estendido para um conjunto de eventos, i.e., $\Psi(\Sigma)=\bigcup_{\sigma \in \Sigma} \Psi(\sigma)$. Provendo-se de um abuso de notação, usa-se $\Sigma_{i} \in s$ para representar que $\bar{s} \cap \Psi\left(\Sigma_{i}\right) \neq \emptyset$, em que $s \in \Sigma^{*}$ e $\Sigma_{i} \subseteq \Sigma$. Uma sequência $s$ é dita de falha se $\Sigma_{f} \in s$; em contrapartida, uma sequência $s$ tal que $\Sigma_{f} \notin s$ é dita normal.

Definição 1. [Diagnosticabilidade (Sampath et al., 1995)] Uma linguagem $L$, prefixo-fechada e viva, é diagnosticável em relação à projeção $P_{o}: \Sigma^{*} \rightarrow \Sigma_{o}^{*}$ e ao conjunto de falhas $\Sigma_{f}$ se:

$$
\begin{gathered}
(\exists z \in \mathbb{N})\left(\forall s \in \Psi\left(\Sigma_{f}\right)\right)(\forall t \in L / s,\|t\| \geq z) \Rightarrow \\
\left(\forall w \in P_{o}^{-1}\left(P_{o}(s t)\right) \cap L\right)\left[\Sigma_{f} \in w\right] .
\end{gathered}
$$

A verificação de diagnosticabilidade por ser realizada por meio de diagnosticadores (Sampath et al., 1995), verificadores (Moreira et al., 2011), entre outros métodos. Um método recentemente proposto na literatura (Viana e Basilio, 2019) utiliza o autômato teste, cujo objetivo é verificar a codiagnosticabilidade de falhas em SEDs. Embora o autômato teste se baseie na construção de um diagnosticador, uma de suas diferenças em relação ao diagnosticador proposto por Sampath et al. (1995) é que ele se baseia na busca por componentes fortemente conexos não triviais $(\mathrm{CFCs})^{1}$, não requer hipóteses relativas à inexistência de

\footnotetext{
1 Um conjunto de estados $B \subseteq X$ é uma CFC de $G$ se: (i) para todo par $x, y \in B, x$ alcança $y$ e vice-versa; (ii) o conjunto $B$ é maximal, i.e., não existe outro estado $z$ que possa ser incluído em $B$ e que satisfaça (i); (iii) se $B=\{x\}(|B|=1$ ), então $\exists \sigma \in \Sigma: f(x, \sigma)=x$.
} 
ciclos de estados conectados por eventos não observáveis e que a linguagem seja viva.

Para construir o autômato teste proposto em Viana e Basilio (2019), primeiro, define-se o autômato rotulador $A_{\ell}=\left(X_{A_{\ell}},\left\{\sigma_{f}\right\}, f_{A_{\ell}}, \Gamma_{A_{\ell}}, x_{0, A_{\ell}}, X_{m, A_{\ell}}\right)$, em que $X_{A_{\ell}}=$ $\{N, Y\}, f_{A_{\ell}}\left(N, \sigma_{f}\right)=f_{A_{\ell}}\left(Y, \sigma_{f}\right)=Y, x_{0, A_{\ell}}=N \mathrm{e}$ $X_{m, A_{\ell}}=X_{A_{\ell}}$. Em seguida, cria-se $G_{\ell}=G \| A_{\ell}=$ $\left(X_{\ell}, \Sigma, f_{\ell}, \Gamma_{\ell}, x_{0, \ell}, X_{m, \ell}\right)$ e constrói-se seu observador $G_{d}=$ $O_{b s}\left(G_{\ell}, \Sigma_{o}\right)=\left(X_{d}, \Sigma_{o}, f_{d}, \Gamma_{d}, x_{0, d}, X_{m, d}\right)$, que modela o diagnosticador. Por fim, o autômato teste é construído da seguinte forma:

$$
G_{C F C}=G_{d} \| G_{\ell}
$$

Teorema 1. (Viana e Basilio (2019)). A linguagem $L$ será diagnosticável em relação a $P_{o}$ e $\Sigma_{f}$ se e somente se não existir nenhuma CFC não triviais composta por estados $\left(x_{d_{1}}, x_{d_{2}}, \ldots, x_{d_{n}}, x_{\ell}\right)$ tais que $x_{d_{i}}$ representem estados incertos e $x_{\ell}$ seja um estado certo.

Note que um estado é dito certo se todas as suas componentes possuem rótulo $\mathrm{Y}$, e um estado é dito incerto se é formado por estados com ambos os rótulos $\mathrm{N}$ e $\mathrm{Y}$.

A preditibilidade (Genc e Lafortune, 2009), é uma propriedade de SEDs que visa determinar se, após dadas observações, sempre é possível ter certeza que um evento $\sigma_{p} \in \Sigma$ irá ocorrer, isto é, todas as sequências que incluem $\sigma_{p}$ possuem prefixos que são únicos. A definição formal de preditibilidade é dada a seguir.

Definição 2. (Preditibilidade (Genc e Lafortune, 2009)). Uma linguagem $L$, prefixo-fechada e viva, é preditível em relação à projeção $P_{o}: \Sigma^{*} \rightarrow \Sigma_{o}^{*}$ e ao conjunto de eventos $\sigma_{p} \in \Sigma_{p}$ se:

$$
(\exists z \in \mathbb{N})\left(\forall s \in \Psi\left(\Sigma_{p}\right)\right)(\exists t \in \bar{s})\left[\left(\Sigma_{p} \notin t\right) \wedge \mathbf{P}\right],
$$

em que $\mathbf{P}:=(\forall u \in L)(\forall v \in L / u)\left[P_{o}(u)=P_{o}(t) \wedge\right.$ $\left.\left(\Sigma_{p} \notin u\right) \wedge(\|v\| \geq z) \Rightarrow\left(\Sigma_{p} \in v\right)\right]$.

A preditibilidade de um sistema pode ser verificada por meio de um observador e "estados preditores", i.e., estados especiais do observador que sucedem a primeira ocorrência de falha em uma sequência e são representados por $X_{p}$. Portanto, uma linguagem $L$ é preditível em relação a $P_{o}$ e $\Sigma_{p}$ se e somente se todos os ciclos alcançados pelos estados $x \in X_{p}$ forem compostos somente por estados certos.

\section{MOTIVAÇÃO DO PROBLEMA}

Nesta seção, serão apresentadas as razões pelas quais é necessária uma abordagem geral para o problema da preditibilidade, em que as hipóteses de ausência de ciclos de estados conectados por eventos não observáveis e linguagem viva, apresentadas em Genc e Lafortune (2009) e Liu (2019), por exemplo, são relaxadas. Em seguida será mostrado por meio de diagnosticadores que a diagnose e a predição são problemas distintos, corroborando o resultado obtido em Kumar e Takai (2010), via verificadores.

Dado um sistema modelado por um autômato $G$, um conjunto de eventos $\Sigma=\Sigma_{o} \dot{\cup} \Sigma_{u o}$, e um evento $\sigma_{p}$ a ser predito, a verificação de preditibilidade por meio de diagnosticadores proposta em Genc e Lafortune (2009) é realizada da seguinte forma: (i) rotula-se o autômato $G$, i.e., $G_{\ell}=G \| A_{\ell}$ (ii) constrói-se o observador de $G_{\ell}$, i.e., $G_{d}$; (iii) definem-se os estados $X_{p}$ de tal forma que sejam

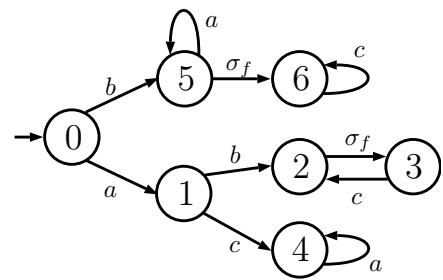

Figura 1. Autômato $G_{1}$

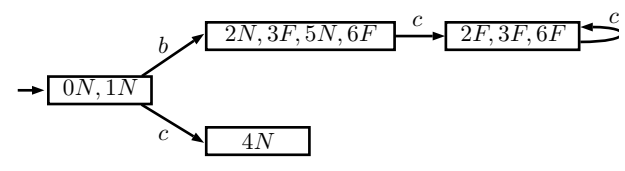

Figura 2. Diagnosticador $G_{d, 1}$

os primeiros estados incertos ou certos precedidos somente por estados normais, e; (iv) verifica-se se todos os estados $X_{p}$ alcançam somente ciclos compostos por estados certos. O sistema será predizível em relação a $P_{o}$ e $\Sigma_{p}$ se e somente se a verificação em (iv) resultar em "sim". Note que só é possível utilizar esse método de verificação supondo-se as seguintes hipóteses antigas como válidas.

HA1. Não há ciclos de estados conectados somente por eventos não observáveis.

HA2. A linguagem gerada pelo sistema é viva.

O exemplo a seguir mostra o efeito de se utilizar a estratégia proposta por Genc e Lafortune (2009) caso a Hipótese HA1 seja violada.

Exemplo 1. Considere um sistema cujo comportamento seja modelado pelo autômato $G_{1}$ apresentado na Figura 1. Suponha que $\Sigma_{o}=\{b, c\}, \Sigma_{u o}=\left\{a, \sigma_{f}\right\}$ e $\Sigma_{f}=\Sigma_{p}=$ $\left\{\sigma_{f}\right\}$. O diagnosticador de $G_{1}$ é $G_{d, 1}=O_{b s}\left(G_{1, \ell}, \Sigma_{o}\right)$, e está representado na Figura 2. Note que nesse diagnosticador todos os ciclos alcançados pelos estados $X_{p}=$ $\{2 N, 3 F, 5 N, 6 F\}$ são compostos por estados certos de falha, o que sugere, conforme o método proposto em (Genc e Lafortune, 2009), que $\sigma_{f}$ é predizível após a observação da sequência $b$. Entretanto, não é difícil verificar que o sistema não é predizível em relação a $\sigma_{f}$, visto que após $s=b$ podem ocorrer inúmeras vezes o evento $a$, sem que $\sigma_{f}$ ocorra. O mesmo acontece para todas as sequências $b a^{*}$, ou seja, não há prefixos das sequências de $\Psi\left(\sigma_{f}\right)$ de tamanho limitado tais que todas suas continuações também sejam de falhas.

Observação 1. Qualquer linguagem não viva $L_{1}$ pode ser transformada em uma outra linguagem viva $L_{2}$ tal que ambas possuam o mesmo comportamento observado, i.e., $P\left(L_{1}\right)=P\left(L_{2}\right)$. Basta adicionar eventos não observáveis $\sigma_{u o} \in \Sigma_{u o}$ em todos as sequências $s$ que não possuem continuação, i.e., adicionar sequencias $s \sigma_{u o}^{*}$ em $L_{1}$.

Uma outra característica ocasionada pela presença de ciclos de estados conectados por eventos não observáveis é que a relação de causa e efeito entre diagnosticabilidade e preditibilidade é afetada, como será ilustrado a seguir.

Exemplo 2. Considere o sistema modelado pelo autômato $G_{1}$, representado na Figura 1 . Conforme apresentado no Exemplo 1, sabe-se que $G_{1}$ não é predizível em relação a $P_{o}$ e $\sigma_{f}$. Por meio do autômato teste de Viana e Basilio (2019), ilustrado na Figura 3, conclui-se que $G_{1}$ é diagnosticável 


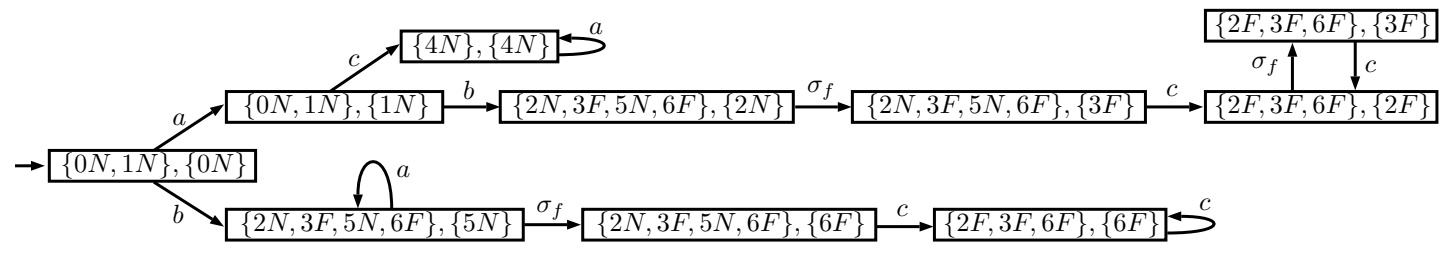

Figura 3. Autômato teste $G_{C F C, 1}$

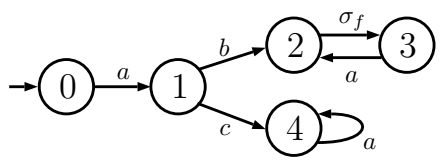

Figura 4. Autômato $G_{2}$

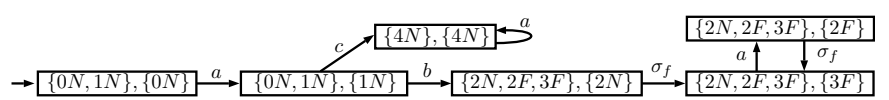

Figura 5. Autômato teste $G_{C F C, 2}$

em relação a $P_{o}$ e $\Sigma_{f}$, visto que não há ciclos compostos por estados em que $x_{d_{i}}$ é incerto sobre a ocorrência de falha e $x_{\ell}$ é rotulado com " $\mathrm{F}$ ".

Exemplo 3. Considere um sistema modelado pelo autômato $G_{2}$, apresentado na Figura 4 , em que $\Sigma_{o}=\{b, c\}$, $\Sigma_{u o}=\left\{a, \sigma_{f}\right\}$ e $\Sigma_{f}=\Sigma_{p}=\left\{\sigma_{f}\right\}$. Note que todas as sequencias de falha $\Psi\left(\Sigma_{f}\right)$ possuem um prefixo comum $s=a b$, e que todas as sequências que possuem observação $b=P_{o}(a b)$ são também prefixos de sequências de falha, ou seja, uma falha eventualmente ocorrerá após observar-se $b, \log$ o, $G_{2}$ é predizível em relação a $P_{o}$ e $\sigma_{f}$. O autômato teste de $G_{2}$, ilustrado na Figura 5, possui um ciclo cujos estados são tais que $x_{d_{i}}$ é incerto e $x_{\ell}$ é rotulado com "F", portanto, $G_{2}$ é não diagnosticável em relação a $P_{o}$ e $\Sigma_{f}$.

Fato 1. Não há relação condicional entre preditibilidade e diagnosticabilidade quando o sistema possui ciclos de estados conectados somente por eventos não observáveis.

O Fato 1 pode ser inferido diretamente de contraexemplos. Dados os autômatos $G_{1}$ e $G_{2}$, apresentados nos Exemplos 2 e 3, pode-se inferir que diagnosticabilidade não é uma condição suficiente para preditibilidade, visto que o sistema modelado por $G_{1}$ é diagnosticável mas não é predizível. Por outro lado, a diagnosticabilidade não é uma condição necessária para preditibilidade, já que o sistema modelado por $G_{2}$ é predizível porém não diagnosticável.

\section{VERIFICAÇÃO DE PREDITIBILIDADE EM SEDS}

Nesta seção são apresentados (i) uma nova estratégia para verificação de preditibilidade, em que as Hipóteses Antigas HA1 e HA2 são relaxadas, e, (ii) um algoritmo inspirado no autômato teste (Viana e Basilio, 2019) para realizar tal verificação.

Conforme apresentado no Exemplo 1 e ilustrado pela Figura 2, sabe-se que ao se construir o diagnosticador $\operatorname{Obs}\left(G_{\ell}, \Sigma_{o}\right)$, a informação sobre ciclos de estados conectados por eventos não observáveis é perdida. A ausência dessa informação impossibilita a verificação de preditibilidade, portanto, uma nova estratégia é necessária.

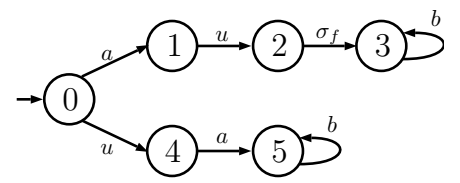

Figura 6. Autômato $G_{3}$

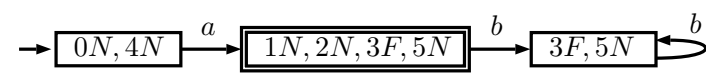

Figura 7. Diagnosticador $G_{d, 3}$

Portanto, este artigo propõe uma estratégia para verificação de preditibilidade que utiliza o autômato teste, o qual permite a visualização de ciclos compostos por eventos não observáveis, e que também utiliza os estados preditores $X_{p}$.

Os estados preditores $x \in X_{p}$ são estados do diagnostica$\operatorname{dor} G_{d}=\operatorname{Obs}\left(G_{\ell}, \Sigma_{o}\right)$ e serão marcados, i.e., $X_{p}=X_{m, d}$. Um estado $x$ é dito preditor, i.e., $x \in X_{p}$, se os estados que o precedem são normais e $x$ é um estado incerto, ou seja, são os primeiros estados incertos alcançados a partir do estado inicial do diagnosticador. Formalmente, $x \in X_{p}$ se e somente se: (i) $x$ é um estado incerto, e (ii) $\forall x^{\prime} \in X_{d}$, $\forall \sigma \in \Sigma_{o}: f_{d}\left(x^{\prime}, \sigma\right)=x, x^{\prime}$ é um estado normal.

Exemplo 4. Seja um sistema cujo comportamento é modelado pelo autômato $G_{3}$ apresentado na Figura 6 , em que $\Sigma_{o}=\{a, b\}, \Sigma_{u o}=\left\{u, \sigma_{f}\right\}$ e $\Sigma_{f}=\Sigma_{p}=\left\{\sigma_{f}\right\}$. O diagnosticador de $G_{3}$ é dado por $G_{d, 3}=O_{b s}\left(G_{3} \| A_{\ell}, \Sigma_{o}\right)$ e ilustrado na Figura 7. Nota-se que $X_{p}=\{1 N, 2 N, 3 F, 5 N\}$, pois este estado é incerto e precedido somente por estados normais, portanto deve-se marcá-lo em $G_{d, 3}$.

A próxima etapa é construir o autômato verificador de preditibilidade. Este verificador é similar ao autômato teste, mas, em sua construção, deve-se levar em conta os estados preditores $X_{p}$. A fim de manter a marcação original referente aos estados preditores $\left(x \in X_{p}\right)$ no verificador, cria-se um autômato $G_{\ell}^{\prime}$, uma cópia de $G_{\ell}$ com todos os seus estados marcados. Definindo-se $G_{C F C}^{\prime}=$ $G_{d} \| G_{\ell}^{\prime}$, os estados cuja primeira componente são estados preditores permanecerão marcados.

Após a construção do verificador, deve-se checar se os ciclos alcançados pelos estados marcados $x \in X_{C F C, m}^{\prime}$ são compostos somente por estados $\left\{x_{d}, x_{\ell}\right\}$ cuja segunda componente $x_{\ell}$ é rotulada por $F$. A linguagem $L$ gerada por um autômato $G$ é preditível em relação a $P_{o}$ e $\Sigma_{p}=$ $\left\{\sigma_{p}\right\}$ se e somente se a resposta da verificação for positiva.

Ao realizar-se a composição paralela entre os autômatos $G_{d}$ e $G_{\ell}^{\prime}$, a marcação de estados, que representam os estados preditores $X_{p}$, é propagada a outros estados do autômato teste $G_{C F C}^{\prime}$ devido à ocorrência do evento $\sigma_{p}$ ser precedida ou sucedida por sequências de eventos não observáveis. Note que, os estados marcados $x \in X_{C F C, m}^{\prime}$ que são 


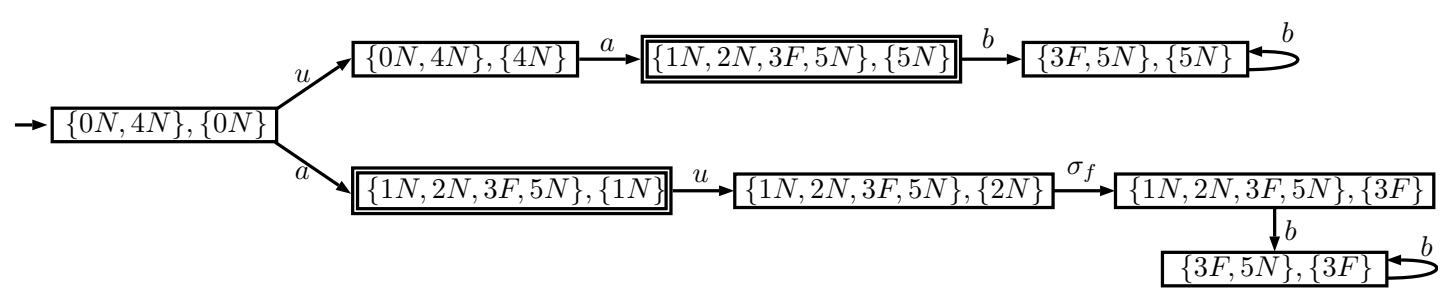

Figura 8. Autômato teste $G_{C F C, 3}$

alcançados por outros estados marcados $y \in X_{C F C, m}^{\prime}$ são na verdade estados preditores redundantes, pois $y$ alcança todos os ciclos que $x$ alcança, e, portanto, os estados $x$ podem ser desmarcados em $G_{C F C}^{\prime}$ sem prejudicar a verificação de preditibilidade. Em suma, o estados marcados não redundantes em $G_{C F C}^{\prime}$ são somente aqueles que são precedidos por eventos observáveis, i.e.,

$$
x=\left\{x_{d}, x_{\ell}\right\} \in X_{C F C, m}^{\prime} \Longleftrightarrow(i) \wedge(i i) \wedge(i i i) .
$$

em que:

(i) $\nexists y \in X_{C F C}^{\prime}, \nexists \sigma_{u o} \in \Sigma_{u o}: f_{C F C}^{\prime}\left(y, \sigma_{u o}\right)=x, y \neq x$;

(ii) $x_{d} \in X_{d, m}$;

(iii) $x_{\ell} \in X_{\ell, m}^{\prime}$.

Exemplo 5. Seja um sistema cujo comportamento é modelado pelo autômato $G_{3}$ apresentado na Figura 6 , em que $\Sigma_{o}=\{a, b\}, \Sigma_{u o}=\left\{u, \sigma_{f}\right\}$ e $\Sigma_{f}=\Sigma_{p}=\left\{\sigma_{f}\right\}$ e cujo diagnosticador $G_{d, 3}$ e ilustrado na Figura 7 . Nota-se que ao construir-se o autômato teste $G_{C F C, 3}^{\prime}=G_{d, 3} \| G_{\ell, 3}^{\prime}$, ilustrado na Figura 8, os estados $\{\{1 N, 2 N, 3 F, 5 N\},\{5 N\}\}$, $\{\{1 N, 2 N, 3 F$,

$5 N\},\{1 N\}\},\{\{1 N, 2 N, 3 F, 5 N\},\{2 N\}\}$ e $\{\{1 N, 2 N, 3 F$, $5 N\},\{3 F\}\}$ serão marcados devido à composição paralela. Entretanto, os estados $\{\{1 N, 2 N, 3 F, 5 N\},\{2 N\}\}$ e $\{\{1 N, 2 N, 3 F, 5 N\},\{3 F\}\}$ são estados preditores redundantes, pois são alcançados por outros estados preditores por meio de eventos não observáveis, e, conforme a Equação 1 , devem ser desmarcados em $G_{C F C, 3}^{\prime}$.

Note que, o estado preditor $x_{d}=\left\{x_{\ell, 1}^{1}, \ldots, x_{\ell, i}^{1}, x_{\ell, 1}^{2}, \ldots\right.$, $\left.x_{\ell, j}^{2}, \ldots, x_{\ell, k}^{n}\right\}$ em $G_{d}$ é incerto, ou seja, por definição, ele representa um conjunto de estados normais $\left\{x_{\ell, 1}^{1}, x_{\ell, 1}^{2}, \ldots, x_{\ell, 1}^{n}\right\}$ e todo seu alcance não observável, que contém estados de falha e podem conter outros estados normais. Além disso, ao realizar-se a composição paralela entre $G_{d}$ e $G_{\ell}^{\prime}$, o número de estados marcados será igual a soma das componentes dos estados preditores, i.e., serão marcados os estados $x_{d} \times x_{\ell, 1}^{1}, \ldots, x_{d} \times x_{\ell, i}^{1}, x_{d} \times x_{\ell, 1}^{2}, \ldots$, $x_{d} \times x_{\ell, j}^{2}, \ldots, x_{d} \times x_{\ell, k}^{n}$. Entretanto, conforme a Equação 1, apenas os estados $x_{d} \times x_{\ell, 1}^{i}, 1 \leq i \leq n$, serão marcados, logo, todos os estados cuja componente $x_{\ell}$ for rotulada por $F$ não serão marcados em $G_{C F C}^{\prime}$. Consequentemente, uma forma simples de reduzir a quantidade de estados redundantes no autômato teste $G_{C F C}^{\prime}$ é marcar somente os estados rotulados por $N$ em $G_{\ell}^{\prime}$.

A definição de estados preditores e estados marcados em $G_{C F C}^{\prime}$ implica diretamente nos dois fatos a seguir:

Fato 2. Toda sequência de falha $s \in \Psi\left(\Sigma_{f}\right)$ no autômato teste $G_{C F C}^{\prime}$ possui um prefixo $t$ em que a falha ainda não ocorreu e que alcança um estado preditor $x \in X_{m, C F C}^{\prime}$, i.e., $\left(\forall s \in \Psi\left(\Sigma_{f}\right)\right),(\exists t \in \bar{s})\left[\left(\Sigma_{f} \notin t\right) \wedge\left(f_{C F C}^{\prime}\left(x_{0, C F C}^{\prime}, t\right) \in\right.\right.$ $\left.\left.X_{m, C F C}^{\prime}\right)\right]$.

Fato 3. Toda sequência normal $u$ com mesma projeção de uma sequência $t$ que leva a um estado preditor $f_{C F C}^{\prime}\left(x_{0, C F C}^{\prime}, t\right) \in X_{m, C F C}^{\prime}$ e que não participa do caminho de $t\left(u \notin \overline{t \Sigma_{u o}^{*}}\right)$ possui um prefixo $u^{\prime}$ com mesma projeção e que também leva a um estado preditor $f_{C F C}^{\prime}\left(x_{0, C F C}^{\prime}, u^{\prime}\right) \in X_{m, C F C}^{\prime}$, i.e., $(\forall u \in$ $\left.L\left(G_{C F C}^{\prime}\right)\right)\left[\left(P_{o}(u)=P_{o}(t)\right) \wedge\left(\Sigma_{f} \notin u\right) \wedge\left(f_{C F C}^{\prime}\left(x_{0, C F C}^{\prime}, t\right) \in\right.\right.$ $\left.\left.X_{m, C F C}^{\prime}\right) \wedge\left(u \notin \overline{t \Sigma_{u o}^{*}}\right)\right] \rightarrow\left(\exists u^{\prime} \in \bar{u}\right)\left[\left(P_{o}(u)=P_{o}\left(u^{\prime}\right)\right) \wedge\right.$ $\left.\left(f_{C F C}^{\prime}\left(x_{0, C F C}^{\prime}, u^{\prime}\right) \in X_{m, C F C}^{\prime}\right)\right]$.

Baseado na estratégia proposta, a verificação de preditibilidade é formulada no teorema a seguir.

Teorema 2. Uma linguagem $L$ é preditível com relação a uma projeção $P_{o}$ e eventos $\Sigma_{p}$ se e somente se os estados marcados $x \in X_{m, C F C}^{\prime}$ no autômato teste $G_{C F C}^{\prime}$ alcançam somente componentes fortemente conexas formadas por estados $\left\{x_{d}, x_{\ell}\right\}$, cuja componente $x_{\ell}$ é rotulada por $F$.

Prova. $(\Leftarrow)$ Suponha que todos os estados marcados $x \in X_{m, C F C}^{\prime}$ em $G_{C F C}^{\prime}$ alcancem somente CFCs formados por estados $\left\{x_{d}, x_{\ell}\right\}$ tal que $x_{\ell}$ seja rotulado com $F$, ou seja, a falha $\sigma_{f}$ eventualmente ocorrerá em todas as continuações das sequências que alcançam os estados $x \in X_{m, C F C}^{\prime}$. Portanto, do Fato 3 segue que $\left(\exists z^{\prime} \in\right.$ $\mathbb{N}),\left(\forall v^{\prime} \in L\left(G_{C F C}^{\prime}\right) / u^{\prime}\right)\left[\left(P_{o}(u)=P_{o}(t)\right) \wedge\left(\Sigma_{f} \notin u\right) \wedge\right.$ $\left.\left(\left\|v^{\prime}\right\| \geq z^{\prime}\right) \rightarrow \Sigma_{f} \in v^{\prime}\right]$, pois a sequência $u^{\prime}$ alcança um estado preditor e, por hipótese, $\sigma_{f}$ ocorrerá em todas suas continuações. Pode-se também inferir que $(\exists z \in \mathbb{N}),(\forall v \in$ $\left.L\left(G_{C F C}^{\prime}\right) / u\right)\left[\left(P_{o}(u)=P_{o}(t)\right) \wedge\left(\Sigma_{f} \notin u\right) \wedge(\|v\| \geq z) \rightarrow\right.$ $\left.\Sigma_{f} \in v\right]$, ou seja, todas as continuações da sequência $u$ também são de falha, pois $u^{\prime} \in \bar{u}$. Deste resultado, do fato de que $L(G)=L\left(G_{C F C}^{\prime}\right)=L$, e do Fato 2 , segue a própria definição de preditibilidade, i.e.

$$
(\exists z \in \mathbb{N})\left(\forall s \in \Psi\left(\Sigma_{p}\right)\right)(\exists t \in \bar{s})\left[\left(\Sigma_{p} \notin t\right) \wedge \mathbf{P}\right],
$$

em que $\mathbf{P}:=(\forall u \in L)(\forall v \in L / u)\left[P_{o}(u)=P_{o}(t) \wedge\right.$ $\left.\left(\Sigma_{p} \notin u\right) \wedge(\|v\| \geq z) \Rightarrow\left(\Sigma_{p} \in v\right)\right]$.

$(\Rightarrow)$ Agora, suponha que exista um estado marcado $x \in$ $X_{m, C F C}^{\prime}$ em $G_{C F C}^{\prime}$ que alcance um CFC formado por estados $\left\{x_{d}, x_{\ell}\right\}$ tal que $x_{\ell}$ é rotulado com $N$, ou seja, existe um caminho após o estado preditor em que a falha $\sigma_{f}$ nunca ocorre. Seja $u$ a sequência que alcança o estado marcado em questão, e, conforme enunciado na definição de estados marcados e nos Fatos 2 e 3, existe uma sequência de falha $s \in \Psi\left(\Sigma_{f}\right)$ cujo prefixo anterior a ocorrência de falha $t \in \bar{s}\left[\Sigma_{f} \notin t\right]$, que alcança um estado marcado $f_{C F C}^{\prime}\left(x_{0, C F C}^{\prime}, t\right) \in X_{m, C F C}^{\prime}$ e possuem mesma projeção $P_{o}(t)=P_{o}(u)$. Portanto, ao se observar 
$P_{o}(t)=P_{o}(u)$, não se pode afirmar que a falha ocorrerá inevitavelmente. Verifica-se, então, se este comportamento acontece para todo prefixo de $s$ anterior à falha, i.e., $\forall t^{\prime} \in \bar{s}\left[\Sigma_{f} \notin t^{\prime}\right]$, que pode ser reescrito como $\bar{t} \cup\left(t\left(\Sigma_{u o}\right)\right.$ $\left.\left.\Sigma_{f}\right)^{*}\right)$. Este comportamento se repete para todo $t^{\prime \prime} \in \bar{t}$, pois existe $u^{\prime \prime} \in \bar{u}$ tal que $P_{o}\left(t^{\prime \prime}\right)=P_{o}\left(u^{\prime \prime}\right)$ e, após esta observação, também não se pode afirmar que a falha ocorrerá inevitavelmente. $\mathrm{O}$ caso das sequências $t^{\prime \prime \prime} \in$ $t\left(\Sigma_{u o} \backslash \Sigma_{f}\right)^{*}$ se reduz ao primeiro caso, visto que $P_{o}\left(t^{\prime \prime \prime}\right)=$ $P_{o}(t)=P_{o}(u)$. Dos resultados obtidos a partir dos Fatos 2 e 3 e da hipótese adotada, segue a negação da definição de predição, i.e.,

$$
(\forall z \in \mathbb{N})\left(\exists s \in \Psi\left(\Sigma_{p}\right)\right)(\forall t \in \bar{s})\left[\left(\Sigma_{p} \notin t\right) \wedge \mathbf{P}\right],
$$

em que $\mathbf{P}:=(\exists u \in L)(\exists v \in L / u)\left[P_{o}(u)=P_{o}(t) \wedge\right.$ $\left.\left(\Sigma_{p} \notin u\right) \wedge(\|v\| \geq z) \Rightarrow\left(\Sigma_{p} \notin v\right)\right]$

A estratégia discutida nesta seção para a verificação de preditibilidade é apresentada no algoritmo a seguir.

\section{Algoritmo 1. (Verificação de preditibilidade).}

Entradas: Autômato $G$.

Saídas: Decisão de preditibilidade: Sim ou Não.

PAsso 1. Construa o autômato rotulado $G_{\ell}$.

PAsso 2. Construa o diagnosticador $G_{d}=O_{b s}\left(G_{\ell}, \Sigma_{o}\right)$.

PAsso 3. Se $X_{p}=\emptyset$, então $L$ não é predizível em relação a $P_{o}$ e $\sigma_{p}=\sigma_{f}$ e o algoritmo responde " $N \tilde{a} o$ ". Caso contrário, marque os estados $X_{p}$ em $G_{d}, X_{m, d} \leftarrow X_{p}$.

PAsso 4. Construa $G_{\ell}^{\prime}=G_{\ell}$ e defina $X_{m, \ell}^{\prime}=\left\{x \in X_{\ell}^{\prime}\right.$ : $x$ possui rótulo $N\}$.

PAsso 5. Se $\exists x \in X_{\ell}^{\prime}: \Gamma_{\ell}^{\prime}(x)=\emptyset$, então defina $f_{\ell}^{\prime}\left(x, \sigma_{u o}\right)=x$, em que $\sigma_{u o} \in \Sigma_{u o}$.

PAsso 6. Construa o autômato teste $G_{C F C}^{\prime}=G_{d} \| G_{\ell}^{\prime}$.

PAsso 7. Para todos os estados $x=\left\{x_{d}, x_{\ell}\right\} \in X_{C F C, m}^{\prime}$ :

$7.1 \mathrm{Se} \exists y \in X_{C F C}^{\prime}, \exists \sigma_{u o} \in \Sigma_{u o}: f_{C F C}^{\prime}\left(y, \sigma_{u o}\right)=x, y \neq$ $x$, então, $X_{C F C, m}^{\prime} \leftarrow X_{C F C, m}^{\prime} \backslash\{x\}$.

PAsso 8. Buscar por todos CFCs não triviais em $G_{C F C}^{\prime}$.

PAsso 9. Verificar se todos os estados marcados $x \in$ $X_{C F C, m}^{\prime}$ alcançam somente CFCs formados por estados $\left\{x_{d}, x_{\ell}\right\}$, em que $x_{\ell}$ é rotulado por $F$.

9.1. Se a resposta da verificação for verdadeira, então $L$ é predizível em relação a $P_{o}$ e $\sigma_{p}=\sigma_{f}$ e o algoritmo responde "Sim". Caso contrário, $L$ não é predizível em relação a $P_{o}$ e $\sigma_{p}=\sigma_{f}$ e o algoritmo responde "Não".

\section{CONCLUSÃO}

Este artigo apresenta uma condição necessária e suficiente para a verificação de preditibilidade em SEDs, assim como uma nova ferramenta para sua verificação que se baseia em um autômato diagnosticador e na busca por componentes fortemente conexos. As hipóteses de linguagem seja viva ou ausência de ciclos de estados conectados por eventos não observáveis não são exigidas.
Futuras direções para esta pesquisa seriam, por exemplo, generalizar a estratégia para estruturas descentralizadas e modulares, e também investigar novas estratégias usando verificadores paras as mesmas hipóteses adotadas.

\section{REFERÊNCIAS}

Cassandras, C.G. e Lafortune, S. (2008). Introduction to Discrete Events Systems. Springer, New York, NY : USA, 2nd edition.

Chen, J. e Kumar, R. (2015). Stochastic failure prognosability of discrete event systems. IEEE Transactions on Automatic Control, 60(6), 1570-1581.

Clavijo, L.B. e Basilio, J.C. (2017). Empirical studies in the size of diagnosers and verifiers for diagnosability analysis. Discrete Event Dynamic Systems, 27(4), 701739.

Genc, S. e Lafortune, S. (2009). Predictability of event occurrences in partially-observed discrete-event systems. Automatica, 45(2), 301-311.

Jiang, S. e Kumar, R. (2004). Failure diagnosis of discreteevent systems with linear-time temporal logic specifications. IEEE Transactions on Automatic Control, 49(6), 934-945.

Khoumsi, A. e Chakib, H. (2009). Multi-decision decentralized prognosis of failures in discrete event systems. In 2009 American Control Conference, 4974-4981. IEEE.

Khoumsi, A. e Chakib, H. (2012). Conjunctive and disjunctive architectures for decentralized prognosis of failures in discrete-event systems. IEEE Transactions on Automation Science and Engineering, 9(2), 412-417.

Kumar, R. e Takai, S. (2010). Decentralized prognosis of failures in discrete event systems. IEEE Transactions on Automatic Control, 55(1), 48-59.

Liu, F. (2019). Predictability of failure event occurrences in decentralized discrete-event systems and polynomialtime verification. IEEE Transactions on Automation Science and Engineering, 16(1), 498-504.

Moreira, M.V., Jesus, T.C., e Basilio, J.C. (2011). Polynomial time verification of decentralized diagnosability of discrete event systems. IEEE Transactions on Automatic Control, 56, 1679-1684.

Sampath, M., Sengupta, R., Lafortune, S., Sinnamohideen, K., e Teneketzis, D. (1995). Diagnosability of discreteevent systems. IEEE Transactions on Automatic Control, 40(9), 1555-1575.

Takai, S. (2015). Robust prognosability for a set of partially observed discrete event systems. Automatica, 51, 123-130.

Takai, S. e Kumar, R. (2012). Distributed failure prognosis of discrete event systems with bounded-delay communications. IEEE Transactions on Automatic Control, 57(5), 1259-1265.

Viana, G.S. e Basilio, J.C. (2019). Codiagnosability of discrete event systems revisited: A new necessary and sufficient condition and its applications. Automatica, 101, $354-364$.

Yin, X. e Li, Z. (2016a). Decentralized fault prognosis of discrete event systems with guaranteed performance bound. Automatica, 69, 375-379.

Yin, X. e Li, Z. (2016b). Reliable decentralized fault prognosis of discrete-event systems. IEEE Transactions on Systems, Man, and Cybernetics: Systems, 46(11), 1598-1603. 